\title{
Functional knee phenotypes: a call for a more personalised and individualised approach to total knee arthroplasty?
}

\author{
Michael T. Hirschmann ${ }^{1,2} \cdot$ Henrik Behrend ${ }^{3}$
}

Received: 21 March 2018 / Accepted: 27 April 2018 / Published online: 10 May 2018

(c) European Society of Sports Traumatology, Knee Surgery, Arthroscopy (ESSKA) 2018

Keywords Total knee arthroplasty $\cdot$ TKA $\cdot$ Total knee replacement $\cdot$ TKR $\cdot$ Knee $\cdot$ Alignment Anatomy $\cdot$ Functional bone morphotypes $\cdot$ Knee phenotypes, hip knee ankle angle $\cdot$ Hip knee angle $\cdot$ Personalized medicine

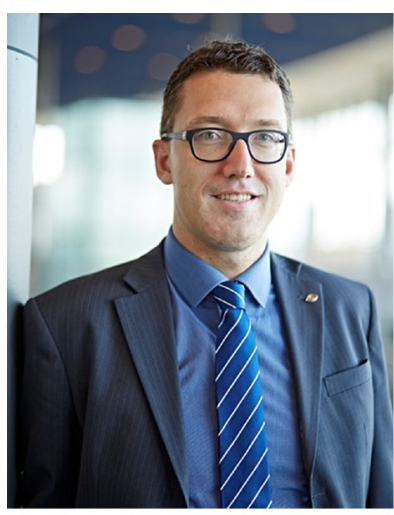

Michael Tobias Hirschmann

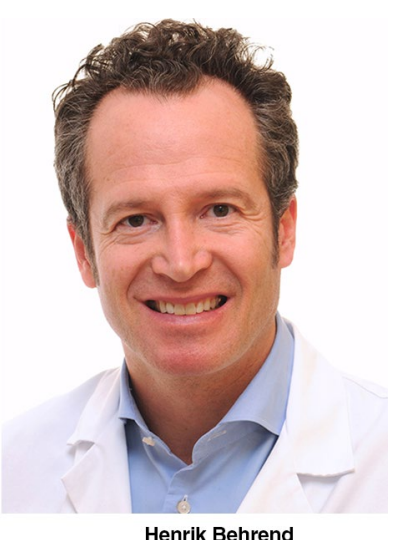

Henrik Behrend
Total knee arthroplasty (TKA) has made major advancements in the past. Landmark steps for improved outcome after TKA were advanced surgical techniques, standardised instrumentation, development of durable material and improved patient care pathways. However, still 20-30\% of patients are not pain free or satisfied after TKA [3]. As expert knee surgeons, we need to ask the question why there is still such a considerable percentage of unhappy patients after TKA. Much effort has already been put into this question by many research groups.

Michael T. Hirschmann

Michael.Hirschmann@unibas.ch

1 Department of Orthopaedic Surgery and Traumatology, Kantonsspital Baselland (Bruderholz, Liestal, Laufen), 4101 Bruderholz, Switzerland

2 University of Basel, Basel, Switzerland

3 Department of Orthopaedic Surgery, Kantonsspital St. Gallen, 9007 St. Gallen, Switzerland
For a long time, a neutral mechanical alignment appeared to be the solution for each knee [5]. Just recently many authors questioned the necessity of neutral mechanical alignment [6, 7]. Bellemans et al. proposed the concept of constitutional alignment for varus knees [1]. Anatomic alignment and kinematic alignment were introduced or reinvented as systematic alignment methods [4]. However, the major problem here is the systematic approach. A systematic approach is based on the theory of a systematic solution for the problem. However, this approach is too simplified. It does not sufficiently consider anatomical variability of each knee. When you treat every knee the same, there will be happy and unhappy knees as some will be in the envelope of function and some will not. Sadly, systematic alignment considerations are not the holy grail of TKA.

Clearly, one of the major misconceptions of TKA nowadays still is that the variability and individuality of each knee are not sufficiently considered in conventional TKA. Neither with regards to alignment nor to morphology, laxity and function.

Although there is a focus on personalised medicine in research nowadays, our knowledge about the variability of each knee is still scarce. The godfathers of knee surgery such as Werner Müller taught us that nothing is so constant as the variability of anatomy [2]. He further told us that a profound understanding of the individuality of each knee is crucial for optimal restoration of the injured knee in reconstructive knee surgery [2]. Clearly, this is also true for knee arthroplasty. It is time to make some steps backwards and meticulously analyse the native and degenerative knee alignment and individual morphology. Due to the improved 3D-CT technology as well as available software, it is the right time to do it $[7,8]$.

This issue of KSSTA contains a number of scientific articles dealing with the question how to improve outcomes 
after TKA. A landmark paper is presented introducing the novel concept of "functional knee phenotypes". This article is based on a large CT series of degenerative knees and opens a window into the distribution of alignment and morphology of degenerative knees. The authors propose that a better understanding of these functional knee phenotypes in each individual patient might help to improve the outcome after TKA. The novelty of this approach to TKA is the combined assessment of preoperative alignment and morphology. Different functional knee phenotypes are described in detail and a concept of classification is proposed.

Only with a better understanding of the individual patient's anatomy and functional morphology, it will be possible to shed light to the unsolved questions in knee arthroplasty. Consequently, a more thorough preoperative 3D analysis of alignment and morphology of each knee needs to be done. Alignment methods, approach as well as type of TKA used need to be tailored to each individual patient. Future will tell if this approach of "functional knee phenotypes" leads to improved outcomes after TKA and holds the promise of fewer unhappy knees after TKA.

Funding There was no external funding used.

\section{Compliance with ethical standards}

Conflict of interest The authors delcare that there is no conflict of interest.

Ethical approval This article does not contain any studies with human participants performed by any of the authors.

Informed consent For this type of study no informed consent is required.

\section{References}

1. Bellemans J, Colyn W, Vandenneucker H, Victor J (2012) The Chitranjan Ranawat award: is neutral mechanical alignment normal for all patients? The concept of constitutional varus. Clin Orthop Relat Res 470(1):45-53

2. Hirschmann MT, Muller W (2015) Complex function of the knee joint: the current understanding of the knee. Knee Surg Sports Traumatol Arthrosc 23(10):2780-2788

3. Hirschmann MT, Testa E, Amsler F, Friederich NF (2013) The unhappy total knee arthroplasty (TKA) patient: higher WOMAC and lower KSS in depressed patients prior and after TKA. Knee Surg Sports Traumatol Arthrosc 21(10):2405-2411

4. Hungerford DS (1995) Alignment in total knee replacement. Instr Course Lect 44:455-468

5. Longstaff LM, Sloan K, Stamp N, Scaddan M, Beaver R (2009) Good alignment after total knee arthroplasty leads to faster rehabilitation and better function. J Arthroplast 24(4):570-578

6. Parratte S, Pagnano MW, Trousdale RT, Berry DJ (2010) Effect of postoperative mechanical axis alignment on the fifteen-year survival of modern, cemented total knee replacements. J Bone Jt Surg Am 92(12):2143-2149

7. Slevin O, Amsler F, Hirschmann MT (2017) No correlation between coronal alignment of total knee arthroplasty and clinical outcomes: a prospective clinical study using 3D-CT. Knee Surg Sports Traumatol Arthrosc 25(12):3892-3900

8. Slevin O, Hirschmann A, Schiapparelli FF, Amsler F, Huegli RW, Hirschmann MT (2017) Neutral alignment leads to higher knee society scores after total knee arthroplasty in preoperatively nonvarus patients: a prospective clinical study using 3D-CT. Knee Surg Sports Traumatol Arthrosc. https://doi.org/10.1007/s0016 7-017-4744-y 